\title{
Wave-induced loads on a lock gate provided with an opening through the ballast tank
}

\author{
Luca Cavallaro $^{1}\left[\right.$ Claudio luppa $^{1} \cdot$ Pietro Scandura $^{1} \cdot$ Enrico Foti $^{1}$
}

Received: 13 May 2020 / Accepted: 15 October 2020 / Published online: 2 November 2020

(c) The Author(s) 2020

\begin{abstract}
The wave loads on a navigation lock gate provided with an opening in the ballast tank are analyzed using a mathematical model based on the linear wave theory. The analysis focuses on the influence of the wave number and the geometrical characteristics of the structure on the applied load. It is shown that the maximum value of the vertical force mainly depends on the height of the ballast tank and on the width of the opening. The wave number for which the maximum load occurs significantly depends on the geometric characteristics of the structure except for the water depth above the ballast tank which has a negligible effect. An increase in the height of the ballast tank causes an increase in the wave load while an increase in the width of the opening causes a decrease in the wave load. Based on the results of the mathematical model an easy to use regression model has been developed which can be employed to evaluate the wave load.
\end{abstract}

Keywords Navigation lock · Sliding gate $\cdot$ Wave load

\section{Introduction}

Lock gates are essential structures of a navigation lock system, since they allow for the retention of water and the locking of vessels above or below the sea level. Several types of lock gates are used around the world: mitre gates, single pivot gates, standing tainter gates, rolling or sliding gates, lift gates, etc.

Rolling and sliding gate for navigation locks are usually equipped with ballast tanks, which allow the load on the roller carriages to be reduced in order to improve the maneuverability. The presence of such elements could produce some disadvantage, among which the increase of vertical force due to waves which may have negative effects especially during the gate movement.

Luca Cavallaro

luca.cavallaro@unict.it

Claudio Iuppa

ciuppa@dica.unict.it

Pietro Scandura

pietro.scandura@unict.it

Enrico Foti

enrico.foti@unict.it

1 Department of Civil Engineering and Architecture, University of Catania, Via Santa Sofia 64, 95123 Catania, Italy
Wave action is often neglected in the design of these structures as they are usually located in waters characterized by small waves with short periods. However, waves might be important for lock gates located along the coastline of sea or great lakes.

The possible negative effects of the wave load on a sliding gate have recently emerged at the seaside gate of the navigation lock realized at the Malamocco inlet of the Venice lagoon, which was designed to allow the access to the Port of Venice during the operative period of the flood control system Mo.S.E. (acronym of 'MOdulo Sperimentale Elettromeccanico' in italian language). The width of such a gate is $50 \mathrm{~m}$ and the height is $16.6 \mathrm{~m}$. The mean water depth in front of the gate is $14 \mathrm{~m}$. During a storm in 2015 the sea-side gate underwent extensive damages due to large vertical oscillations because of waves characterized by height and mean period equal to about $1 \mathrm{~m}$ and $8 \mathrm{~s}$ respectively.

An analysis of the wave loads on a lock gate similar to that at the Malamocco inlet was recently performed by Cavallaro et al. (2018), in which the gate was modelled as a totally immersed ballast tank with a vertical wall adjacent to it. It was observed that the vertical forces are mainly due to the difference in pressure between the upper and lower side of the ballast tank. In order to reduce the vertical forces several new designs of the gate were proposed. The gate which appears to cause lower vertical forces on the ballast tank is equipped 
with a front plate and a large vertical opening which connects the upper and lower side of the ballast tank. In the Malamocco gate, the connection between the upper and lower sides of the ballast tank was obtained by means of vertical tubes (see Fig. 1).

The loads induced by the waves on a similar structure have not yet been analyzed in detail and in the literature there are no formulas or diagrams available for engineering purposes.

Therefore, the forces produced by the waves on these structures could only be assessed through complex physical or numerical models that are not of practical use during the decision-making or pre-design phase of a work. To fill this gap, easy-to-use analytical models and/or empirical approaches should be developed to evaluate optimal design parameters in the first phase of a project (Liu and Li 2013; Monk et al. 2013).

The analytical models used to describe the complex wavestructure interaction generally employ linear potential theory and the eigenfunction expansion matching method to solve the governing equations. This is a classic method to analyse the interaction between waves and structures that has been employed in several studies. Using this approach Losada et al. (1992) studied the interaction between waves and three different types of vertical thin barriers: a descending barrier from the water surface to a given depth; an underwater barrier and a vertical barrier extending from the bottom to the surface with an horizontal slit. Results about the transmission and reflection coefficients were in a good agreement with previous theoretical and experimental studies. Losada et al. (1993) extended the previous work to wave group finding that maximum short wave reflection is associated with maximum long wave transmission of the free long wave component. In Jalón et al. (2018) the same approach was used to study the interaction of waves with coastal structures taking into account the resistances. Guo et al. (2015) adopted the potential flow approach to study the wave forces acting on semi-submerged bridge decks while Fang et al. (2018) solved the same problem for the case of oblique wave attack.

Other problems related to wave-structure interaction analyzed by means of the velocity potential approach are related to elastic floating plates (Wu et al. 1995), a group of submerged horizontal plates (Wang and Shen 1999), two layers of horizontal thick plates (Liu et al. 2009), and oblique scattering of gravity waves by moored floating membrane (Karmakar and Soares 2012; Behera et al. 2018; Kundu et al. 2018). This approach has also been used by Malara and Arena (2013) to investigate the performance of a wave energy converter.

In the present paper, the wave load acting on a gate with a front plate and a prism-shaped ballast tank provided with a large vertical opening is studied by means of the linear wave theory. In Sect. 2, the mathematical formulation of the boundary value problem is shown and the eigenfunction expansion method is adopted to determine the analytical solution. In Sect. 3, the analytical solution is used to analyse the wave force induced on the gate for different geometrical configurations and hydrodynamic conditions. In Sect. 4 an empirical method to evaluate the maximum vertical force induced by waves on a gate is presented. Finally, in Sect. 5 the conclusions are drawn.

\section{Formulation of the problem and analytical solution}

The cross section of the navigation lock is shown in Fig. 2 where the position of the reference system $(x, y, z)$ and the direction of the incoming waves are also highlighted. The geometric parameters that characterize the flow around the structure are: (i) the water depth above the ballast tank $a$; (ii) the width of the gate $b$; (iii) the width of the vertical connection through the ballast tank $c$; (iv) the height of the gap at the bottom of the frontal panel $d$; (v) the height of the ballast tank $s$; (vi) the water depth in front of the gate $h$; (vii) the thickness $s_{1}$ of the front plate. In addition, the hydrodynamic parameters involved in the analysis are the incident wave height $H$ and the wavelength $L$. It is assumed that the waves and the gate have constant geometrical characteristics along the $y$ axis of the reference system so that the flow can be assumed as two-dimensional.

The flow field generated by progressive waves propagating towards the navigation lock is determined following a procedure analogous to that described in Cavallaro et al. (2018). Assuming that the incoming wave is sinusoidal and has an amplitude $H / 2$ much smaller than the wavelength $L$, the linear wave theory can be applied. Therefore, after writing the potential as $\Phi(x, z, t)=\phi(x, z) e^{i \sigma t}$, where $i$ is the imaginary unit, $\sigma$ is the angular frequency and $\phi$ is a complex potential function, the Laplace equation and boundary conditions shown below must be satisfied:

$$
\begin{aligned}
& \frac{\partial^{2} \phi}{\partial x^{2}}+\frac{\partial^{2} \phi}{\partial z^{2}}=0 \\
& \frac{\partial \phi}{\partial \mathbf{n}}=0 \quad \text { at the rigid boundaries, } \\
& -\sigma^{2} \phi+g \frac{\partial \phi}{\partial z}=0 \quad \text { at } z=0
\end{aligned}
$$

where $g$ is the gravity acceleration and $\mathbf{n}$ is the unit vector normal to the rigid boundaries. The pressure $p$ is determined through the following relation:

$p=-\operatorname{Re}\left(i \sigma \varrho \phi e^{i \sigma t}\right)$

where $\rho$ is the fluid density and $R e$ denotes the real part of a complex number. The correctness of using the poten- 
Fig. 1 3D sketches of lock gates with possible vertical openings in the ballast tank. The structural rack is omitted. a Rectangular large opening; (b) vertical tubes

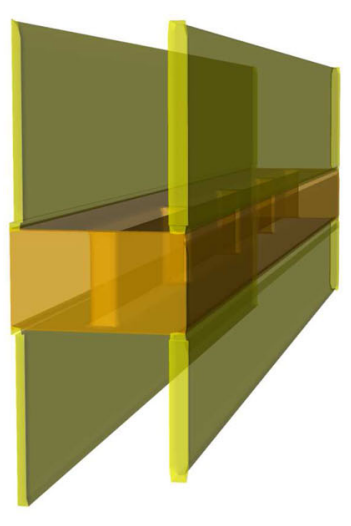

(a)
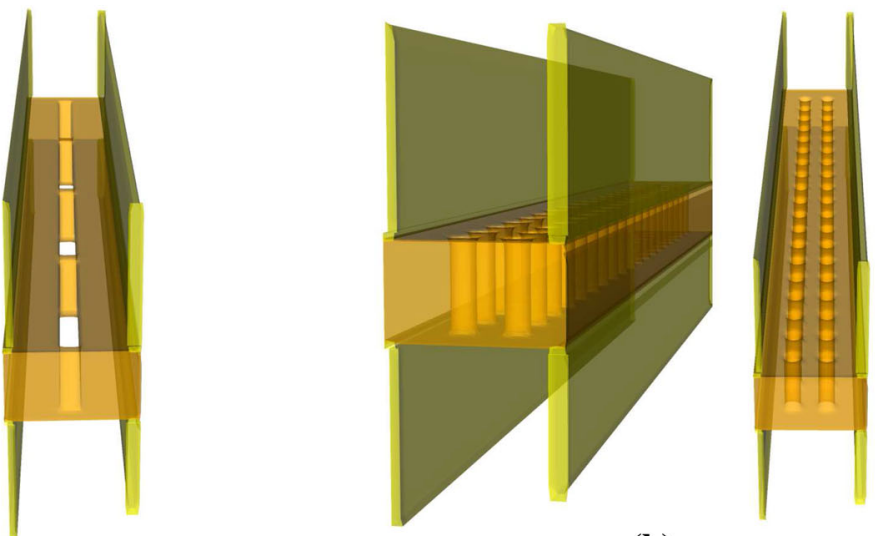

(b) tial theory in this context is due to the oscillatory nature of the wave induced flow. Under these conditions the order of magnitude of the thickness of the viscous boundary layer generated at the rigid boundaries is given by $\sqrt{2 v / \sigma}$ where $v$ is the kinematic viscosity. Considering periods of few seconds and the kinematic viscosity of water, the thickness of the viscous boundary layer turns out to be of the order of millimeters. This thickness is very small compared to the size of the structure, so the flow can be assumed as irrotational in most of the fluid domain.

The mathematical problem posed by Eqs. (1-3) has been solved by means of the eigenfunctions expansion matching method. The fluid domain has been divided into seven regions $\left(\Pi_{1}, \Pi_{2}, \ldots ., \Pi_{7}\right)$ as shown in Fig. 2 . The region $\Pi_{2}$, located below the plate, has been also introduced in order to be able to treat even those cases in which the thickness $s_{1}$ of the plate is not small. The expressions of the potential function in each of the seven sub-domains are shown below (Eqs. 5-11):

$$
\begin{aligned}
\phi_{1}= & \frac{H}{2} \frac{g}{\sigma} \frac{\cosh \left[k_{1}(z+h)\right]}{\cosh \left(k_{1} h\right)} e^{-i k_{1} x} \\
& +A_{11} \cos h\left[k_{1}(z+h)\right] e^{i k_{1} x} \\
& +\sum_{n=2}^{N_{1}} A_{1 n} \cos \left[k_{n}(z+h)\right] e^{k_{n} x} \\
\phi_{2}= & A_{21}+B_{21} x+\sum_{n=2}^{N_{2}} \cos \left[\delta_{n}(z+h)\right] \\
& \left(A_{2 n} e^{\delta_{n} x}+B_{2 n} e^{-\delta_{n} x}\right) \\
\phi_{3}= & A_{31}+B_{31} x+\sum_{n=2}^{N_{3}} \cos \left[\beta_{n}(z+h)\right] \\
& \left(A_{3 n} e^{\beta_{n} x}+B_{3 n} e^{-\beta_{n} x}\right) \\
\phi_{4}= & \cosh \left[k_{1}(z+h)\right]\left(A_{41} e^{i k_{1} x}+B_{41} e^{-i k_{1} x}\right) \\
& +\sum_{n=2}^{N_{4}} \cos \left[k_{n}(z+h)\right]\left(A_{4 n} e^{k_{n} x}+B_{4 n} e^{-k_{n} x}\right)
\end{aligned}
$$

$$
\begin{aligned}
\phi_{5}= & A_{51}+\sum_{n=2}^{N_{5}} A_{5 n} \\
& \cos \left[\beta_{n}(z+h)\right] \cosh \left(\beta_{n}\left(x-s_{1}-b\right)\right) \\
\phi_{6}= & A_{61} \cosh \left[\lambda_{1}(z+a)\right] \cos \left[\lambda_{1}\left(x-s_{1}\right)\right] \\
& +\sum_{n=2}^{N_{6}} A_{6 n} \cos \left[\lambda_{n}(z+a)\right] \cosh \left[\lambda_{n}\left(x-s_{1}\right)\right] \\
\phi_{7}= & A_{71} \cosh \left[\lambda_{1}(z+a)\right] \cos \left[\lambda_{1}\left(x-s_{1}-b\right)\right] \\
& +\sum_{n=2}^{N_{7}} A_{7 n} \cos \left[\lambda_{n}(z+a)\right] \cosh \left[\lambda_{n}\left(x-s_{1}-b\right)\right]
\end{aligned}
$$

where $A_{j n}$ and $B_{j n}$ are unknown expansion coefficients. In the region 1 , the potential is constituted by the sum of three contributions. The first is due to the potential of the incident waves characterized by an amplitude $H / 2$ and frequency $\sigma$. The second is due to the potential of waves reflected from the structure, therefore they travel in the negative $x$ direction. The third is due to the sum of several evanescent waves, in fact their amplitudes decay exponentially with the distance from the structure. In the regions 2, 3 and 5 the potential is given by a contribution constant along the depth plus a contribution variable along the depth. It easy to check that in these regions the overall flow rate only depends on the first contribution. Finally, in domains 4, 6 and 7 the potential is due to waves travelling in both the positive and the negative $x$ directions plus potentials due to waves that vary exponentially in the $x$ direction.

The wavenumbers $k_{n}$ (see Eqs. 5 and 8) satisfy the following dispersion relations:

$\begin{aligned} \sigma^{2} & =g k_{n} \tanh \left(k_{n} h\right) n=1 \\ \sigma^{2} & =-g k_{n} \tan \left(k_{n} h\right) n>1\end{aligned}$

The wavenumbers $\lambda_{n}$ still satisfy Eqs. $(12,13)$ with $h$ replaced by $a$ (see Fig. 2). Finally the wavenumbers $\delta_{n}$ and 


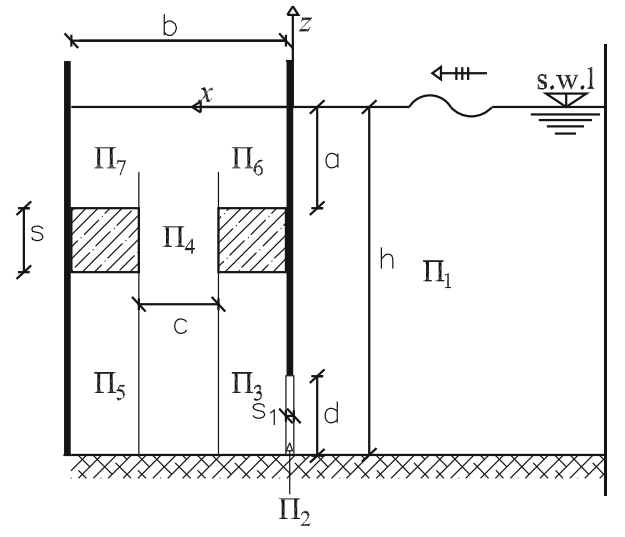

Fig. 2 Transverse section of the lock gate. Water depth above the ballast tank $a$; width of the lock gate $b$; width of the vertical opening in the ballast tank $c$; height of the bottom opening $d$; height of the ballast tank $s$; thickness of the front plate $s_{1}$; water depth $h$

$\beta_{n}$ are computed as follows:

$\delta_{n}=\frac{(n-1) \pi}{d}$

$\beta_{n}=\frac{(n-1) \pi}{h_{1}}$

where $h_{1}=h-(a+s)$.

Equations (13-15) provide an infinite number of values for $k_{n}, \delta_{n}, \beta_{n}$ and $\lambda_{n}$ but only a finite number of terms are retained in the expansion series shown in Eqs. (5-11), so that the total number $N_{t}$ of unknown coefficients is $2\left(N_{2}+N_{3}+\right.$ $\left.N_{4}\right)+N_{1}+N_{5}+N_{6}+N_{7}$. In particular, the number of $A_{j n}$ coefficients is $\sum_{i=1}^{7} N_{i}$, while the number of $B_{j n}$ coefficients is $\sum_{i=2}^{4} N_{i}$.

At the interface between two sub-domains the velocity and the pressure computed on the basis of the potential funcion $\phi_{j}$, pertinent to each of the two subdomains, must provide the same value. Imposing this condition for all sub-domains that share a part of their boundary a linear system of $N_{t}$ equations is obtained whose solution provides the $A_{j n}$ and $B_{j n}$ expansion coefficients. The procedure for obtaining this linear system is rather repetitive, therefore it is sufficient to show it for the subdomains $\Pi_{1}$ and $\Pi_{2}$.

First of all along the left boundary of subdomain $\Pi_{1}$ the continuity of the velocity field imposes the boundary conditions:

$$
\begin{aligned}
& \frac{\partial \phi_{1}}{\partial x}=\frac{\partial \phi_{2}}{\partial x}-h \leq z \leq-(h-d) \\
& \frac{\partial \phi_{1}}{\partial x}=0-(h-d) \leq z \leq 0
\end{aligned}
$$

Equations (16-17) are multiplied by $\cos \left[k_{n}(z+h)\right]$ (by $\cosh \left[k_{n}(z+h)\right]$ for $\left.n=1\right)$, which is the eigenfunction that provides the structure of the solution along the vertical direc- tions in sub-domain $\Pi_{1}$. Then, these equations are integrated over the respective domains of definition and added together to get the following result:

$$
\begin{aligned}
& \int_{-h}^{0} \frac{\partial \phi_{1}}{\partial x} \cos \left[k_{n}(z+h)\right] d z \\
& \quad=\int_{-h}^{-(h-d)} \frac{\partial \phi_{2}}{\partial x} \cos \left[k_{n}(z+h)\right] d z
\end{aligned}
$$

By varying $n$ from 1 to $N_{1}$ and making use of the orthogonality properties of the functions $\cos \left[k_{n}(z+h)\right]$ in the range $(-h, 0), N_{1}$ independent equations are obtained.

Further equations are obtained by imposing that the pressure must be continuous across the boundary shared by two sub-domains. Recalling Eq. (4) this translates in a continuity condition for the potential function. The matching of the potential function along the boundary between regions $\Pi_{1}$ and $\Pi_{2}$ is written as

$\phi_{2}=\phi_{1} \quad-h \leq z \leq-(h-d)$

Equation (19) is multiplied by $\cos \left[\delta_{n}(z+h)\right]$, which is the eigenfunction along the $z$ direction in sub-domain $\Pi_{2}$, and then it is integrated in the interval $-h \leq z \leq-(h-d)$ as follows:

$$
\begin{aligned}
& \int_{-h}^{-(h-d)} \phi_{2} \cos \left[\delta_{n}(z+h)\right] d z \\
& \quad=\int_{-h}^{-(h-d)} \phi_{1} \cos \left[\delta_{n}(z+h)\right] d z
\end{aligned}
$$

Once again, letting $n$ vary from 1 to $N_{2}$ and making use of the orthogonal properties of the functions $\cos \left[\delta_{n}(z+h)\right]$ in the interval $-h \leq z \leq-(h-d), N_{2}$ independent equations are obtained.

Proceeding for all the shared boundaries between subdomains as previously illustrated, a systems of $N_{t}$ equations in the $N_{t}$ unknown coefficients is obtained. In this linear system the non vanishing known terms derive from the first term in Eq. (5), which represents the potential of the incoming wave. The solution of this linear system has been carried out by means of the Gauss elimination approach. The number of expansion coefficients for each subdomain used in the present computations ranges from 30 to 100 while the total number of unknown coefficients $N_{t}$ ranges from 400 to 600 . After computing the expansion coefficients for fixed characteristics of the structure and of the incident wave, the correctness of the procedure was checked by verifying that all the matching conditions along the boundary of each subdomain were satisfied both for the pressure and the velocity.

The vertical force applied on the navigation lock arises from the pressure acting on the lower and upper faces of the ballast tank. Therefore, the solutions in the regions 
$\Pi_{3}, \Pi_{5}, \Pi_{6}$ and $\Pi_{7}$ must be considered to compute the vertical force. The contributions to the vertical force due to the flow in these subdomains are expressed as follows:

$$
\begin{aligned}
f_{3}=- & \varrho i \sigma \frac{A_{31} b_{1}\left(b_{1}+2 s_{1}\right)}{2} \\
& -\varrho i \sigma B_{31} b_{1}-\varrho i \sigma \sum_{n=2}^{N} \frac{\cos \left(\beta_{n} h_{1}\right)}{\beta_{n}}\left[A_{3 n} e^{\beta_{n} s_{1}}\right. \\
& \left.\left(-1+e^{\beta_{n} b_{1}}\right)+B_{3 n} e^{-\beta_{n} s_{1}}\left(1-e^{-\beta_{n} b_{1}}\right)\right] \\
f_{5}=- & \varrho i \sigma A_{51}\left(b-b_{2}\right) \\
& -\varrho i \sigma \sum_{n=2}^{N} \frac{A_{5 n} \cos \left[\beta_{n} h_{1}\right]}{\beta_{n}} \sinh \left[\beta_{n}\left(b-b_{2}\right)\right] \\
f_{6}=\varrho i \sigma A_{61} \frac{\sin \left(\lambda_{1} b_{1}\right)}{\lambda_{1}} & \\
& +\varrho i \sigma \sum_{n=2}^{N} A_{6 n} \frac{\sinh \left(\lambda_{n} b_{1}\right)}{\lambda_{n}} \\
f_{7} & =\varrho i \sigma A_{71} \frac{\sin \left[\lambda_{1}\left(b-b_{2}\right)\right]}{\lambda_{1}} \\
& +\varrho i \sigma \sum_{n=2}^{N} A_{7 n} \frac{\sinh \left[\lambda_{n}\left(b-b_{2}\right)\right]}{\lambda_{n}}
\end{aligned}
$$

where $b_{1}=(b-c) / 2$ and $b_{2}=(b+c) / 2$. The total vertical force $F_{v}$ acting on the navigation lock per unit of length in $y$ direction is given by $F_{v}=f_{3}+f_{5}+f_{6}+f_{7}$. This force can be written as: $F_{v}=\left|F_{v}\right| e^{i \alpha}$, where $\left|F_{v}\right|$ is the amplitude of the force and $\alpha$ denotes the phase. How $\left|F_{v}\right|$ and $\alpha$ depend on the wave and on the navigation lock geometrical parameters is shown in the Sect. 4.

\section{Validation of the model}

In the literature, there are no results of previous studies on the wave load acting on structures similar to that shown in the Fig. 2, to be used for comparison with the results of the present model. The structure considered in Zheng et al. (2004), shown in the Fig. 3, has similarities with the current one in that it can be studied by means of the present model by appropriately choosing the geometric parameters shown in Fig. 2.

The structure of Zheng et al. (2004) consists in a rectangular caisson placed close to a vertical wall. It has a bottom opening, which is the space occupied by subdomain 2 with height denoted as $d$ in Fig. 3, but it is not provided with a ballast tank. Nevertheless, a dummy ballast tank has been drawn with thin lines in Fig. 3. It is clear that the structure considered by Zheng et al. (2004) can be obtained letting the opening $c$ approaching $b$ as this makes the volume occupied by the ballast tank to vanish. For the verification, the

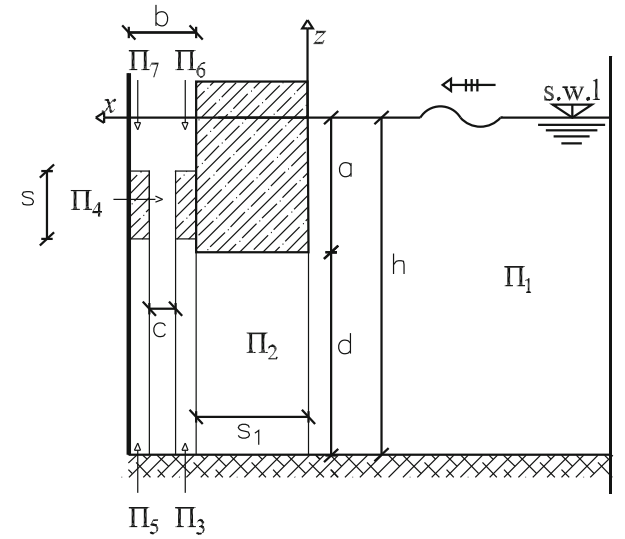

Fig. 3 Sketch of the domain adopted to reproduce Zheng et al. (2004) results. Note that the ballast tank drawn with thin lines is not present in the structure considered by Zheng et al. (2004)

dimensionless parameters of the structure have been fixed as follows: $h / s_{1}=3,(h-d) / h=0.4, b / h=0.2, s / h=0.2$ and computations of the horizontal and vertical forces acting on the caisson have been carried for $c / b=0.4,0.6,0.8,1$.

The results shown by Zheng et al. (2004) were obtained by applying both the eigenfunction expansion method and a boundary element method obtaining indistinguishable results between themselves. Figure 4 shows the dimensionless amplitude of the horizontal $2\left|F_{h}\right| /\left(\varrho g s_{1} H\right)$ and vertical $2\left|F_{v}\right| /\left(\varrho g s_{1} H\right)$ forces versus $h / L$ reported by Zheng et al. (2004) and those computed by means of the present model for the values of $c / b$ previously specified. It can be observed that as $c / b$ tends to 1 present results become indistinguishable from those of Zheng et al. (2004). Although this verification was carried on the basis of results relating to a structure not equipped with a ballast tank, it provides us with strong indications that present analytical solution is correct mainly for two reasons: the model included the presence of a ballast tank with thickness $s$, even if of zero volume when $c / b=1$; the force acting on the caisson is continuous when $c / b$ approaches 1 .

\section{Discussion of model results}

This section shows the results relating to the effect of geometric and hydrodynamic parameters on the vertical force acting on the gate.

According to the Buckingham theorem, the relation among the vertical force, $F_{v}$, acting on the gate per unit width in the $y$ direction and the geometric and hydrodynamic parameters that affect the flow can be written in dimensionless form as follows:

$\left|f_{v}^{*}\right|=\frac{\left|F_{v}\right|}{\rho g H(b-c)}=\psi\left(\frac{a}{h}, \frac{b}{h}, \frac{c}{h}, \frac{d}{h}, \frac{s}{h}, k h, \frac{H}{h}\right)$ 
Fig. 4 Comparison between the amplitude of the dimensionless force reported by Zheng et al. (2004) and that computed by the present model for $h / s_{1}=3$, $(h-d) / h=0.4, b / h=0.2$ and $c / h=b / h$ : (a) vertical force; (b) horizontal force

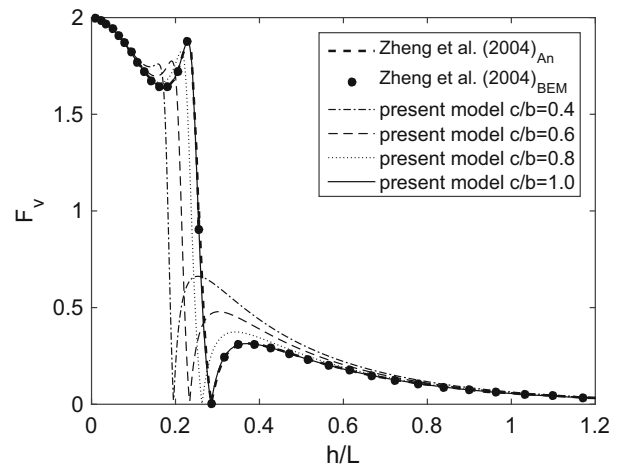

(a)

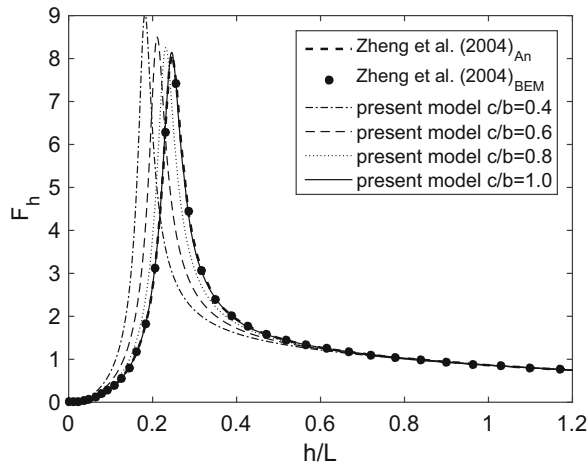

(b)
Table 1 Range of the dimensionless geometrical parameters considered in the study

\begin{tabular}{lll}
\hline Geometrical parameter & Min & Max \\
\hline$a / h$ & 0.286 & 0.429 \\
$b / h$ & 0.286 & 0.571 \\
$c / h$ & 0.057 & 0.171 \\
$d / h$ & 0.071 & 0.214 \\
$s / h$ & 0.143 & 0.429 \\
\hline
\end{tabular}

where $\left|f_{v}^{*}\right|$ is the dimensionless force and $\psi$ is a function of the dimensionless parameters. Since the number of parameters that affect the force is relatively large, studying the function (25) across all the parameter space is not feasible. Therefore, it is necessary to limit the range of parameters so as to find an appropriate compromise between the need to provide a broad picture of the behavior of the function (25) and that of not incurring in non-economic computational commitments. Table 1 shows the range of dimensionless parameters considered in this study.

The vertical force $\left|f_{v}^{*}\right|$ can be expressed as the sum of the force acting on the upper face $f_{v \text { sup }}^{*}$ and on the lower face $f_{v i n f}^{*}$ of the ballast tank. These forces have a phase difference $\Delta \alpha$ equal to zero or equal to $\pi$ radians, depending on $k h$ and the geometrical parameters.

Figures 5, 6, 7, 8, 9 show the dimensionless total vertical force $\left|f_{v}^{*}\right|$ acting on the ballast tank, the forces acting on the upper and lower face of the ballast tank and their phase versus the wave number $k h$ for some geometrical configurations.

In general, it is observed that the total force acting on the ballast tank and the forces acting on the lower and upper face show a maximum for a wave number $k h$ depending on the geometric characteristics of the gate.

Figure 5 shows that the relative depth above the ballast tank $(a / h)$ has a negligible influence on the dimensionless vertical force $\left|f_{v}^{*}\right|$. Even the forces acting on the lower and the upper face of the ballast tank and the phases are weakly affected by the water depth $a$. (a)

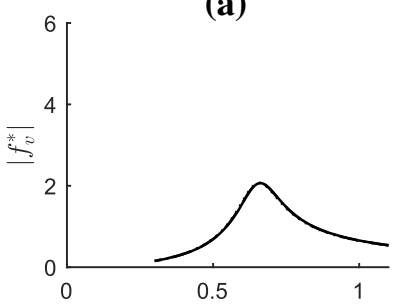

(c)
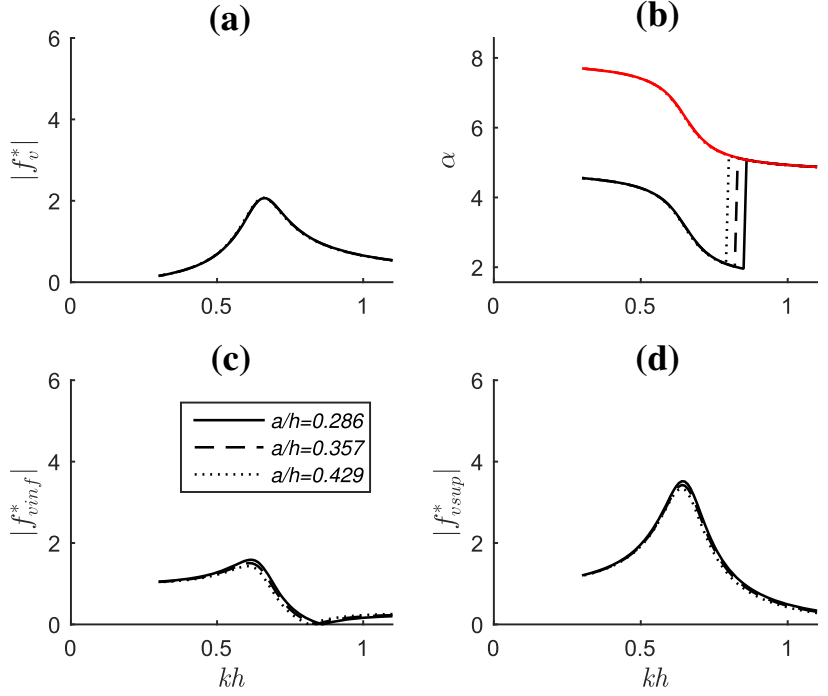

(d)

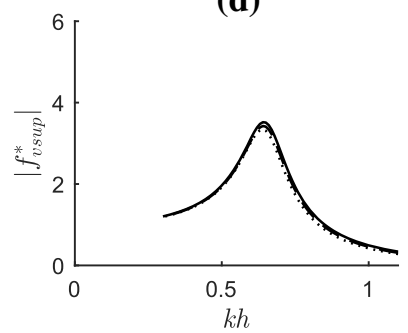

Fig. 5 Amplitude and phase of the force acting on the ballast tank versus $k h$ for $b / h=0.429, c / h=0.114, d / h=0.143$ and $s / h=0.286$ for different values of $a / h$ : (a) amplitude of the total vertical force; (b) phase of the vertical force on the upper face (red lines) and on the lower face (black lines); (c) amplitude of the vertical force on the lower face; (d) amplitude of the vertical force on the upper face

In Figure 6 it can be observed that the maximum force slightly decreases as $b / h$ increases while the value of $k h$ at which the maximum force occurs shifts to lower $k h$. Overall, Fig. 6 shows that the parameter $b / h$ plays an important role in determining the force characteristics.

Figure 7 shows a significant effect of $c / h$ on the force. An increase of $c / h$ causes a decrease of the maximum value of $\left|f_{v}^{*}\right|$ and the shift of the maximum towards larger $k h$. For example, when $c / h$ increases from 0.057 up to 0.171 , the maximum of the force is halved. This effect is a measure of the benefit of introducing the opening through the ballast tank.

Figure 8 shows that, in the range of parameters here analyzed, $d / h$ does not have a very important effect both on the maximum of the vertical force and on the value of $k h$ at which it occurs. An increase of $d / h$ causes an increase of the 

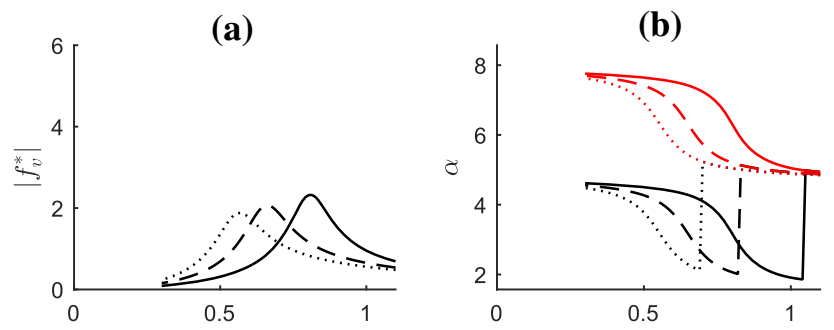

(c)
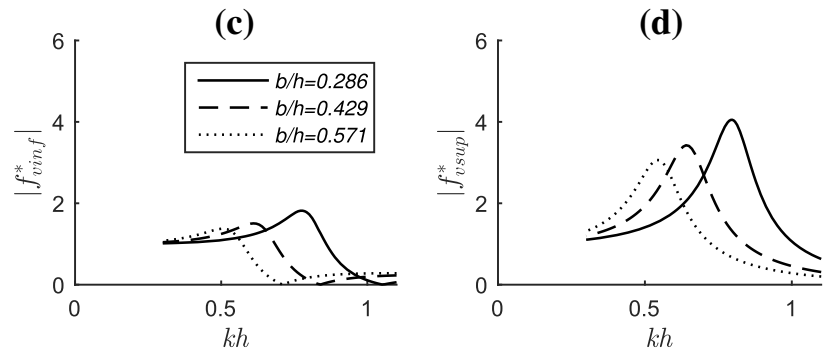

Fig. 6 Amplitude and phase of the force acting on the ballast tank versus $k h$ for $a / h=0.357, c / h=0.114, d / h=0.143$ and $s / h=0.286$ for different values of $b / h$ : (a) amplitude of the total vertical force; (b) phase of the vertical force on the upper face (red lines) and on the lower face (black lines); (c) amplitude of the vertical force on the lower face; (d) amplitude of the vertical force on the upper face (a)

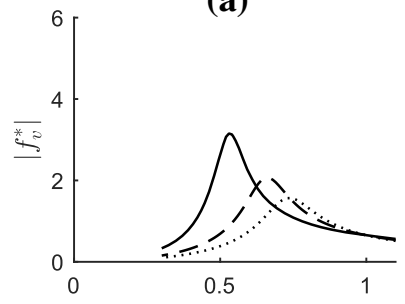

(c)

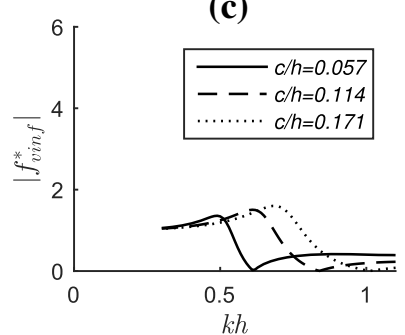

(b)

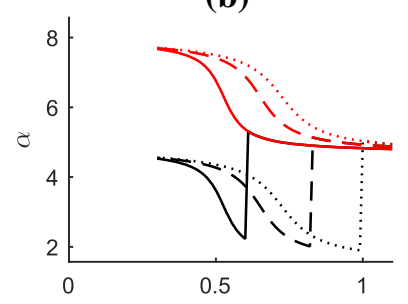

(d)

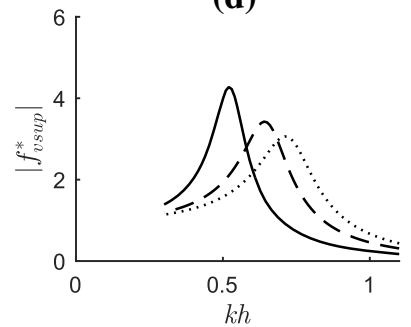

Fig. 7 Amplitude and phase of the force acting on the ballast tank versus $k h$ for $a / h=0.357, b / h=0.429, d / h=0.143$ and $s / h=0.286$ for different values of $c / h$ : (a) amplitude of the total vertical force; (b) phase of the vertical force on the upper face (red lines) and on the lower face (black lines); (c) amplitude of the vertical force on the lower face; (d) amplitude of the vertical force on the upper face

vertical force and the displacement of the maximum towards higher values of $\mathrm{kh}$.

Figure 9 shows that a great effect on vertical force is exerted by the parameter $s / h$ which causes an increase of the force when $s / h$ increases and the displacement of the maximum towards lower values of $k h$. The increase of the force with $s / h$ is explained considering that the force acting (a)

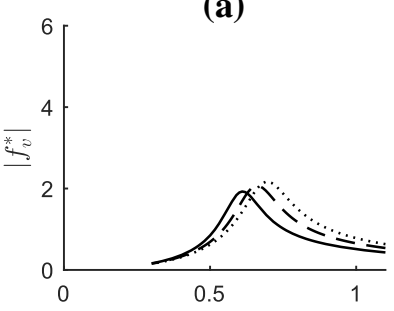

(c)
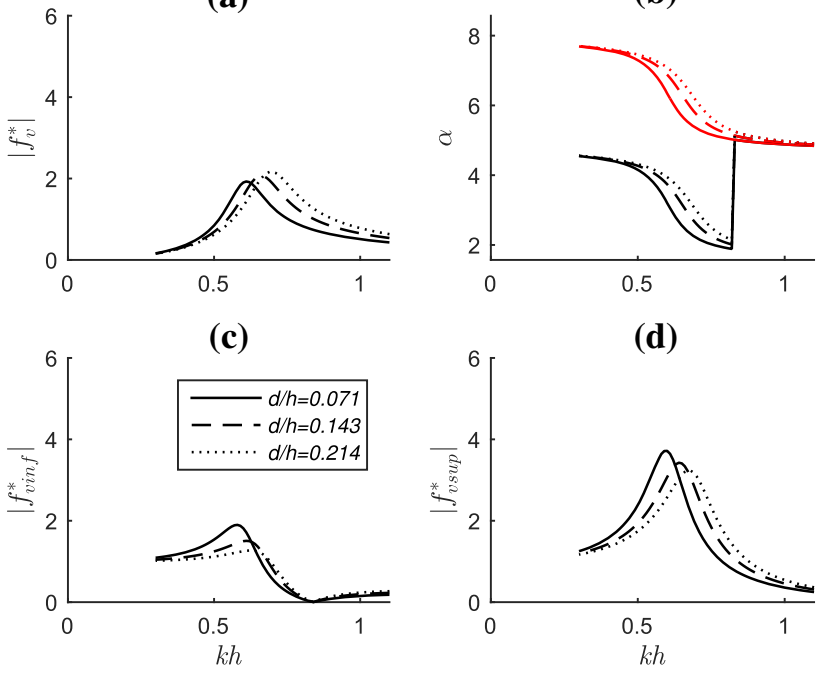

(d)

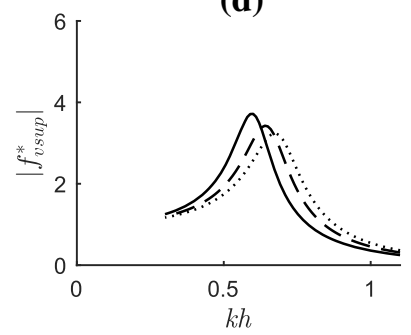

Fig. 8 Amplitude and phase of the force acting on the ballast tank versus $k h$ for $a / h=0.357, b / h=0.429, c / h=0.114$, and $s / h=0.286$ for different values of $d / h$ : (a) amplitude of the total vertical force; (b) phase of the vertical force on the upper face (red lines) and on the lower face (black lines); (c) amplitude of the vertical force on the lower face; (d) amplitude of the vertical force on the upper face

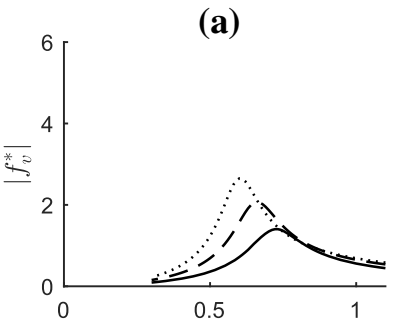

(c)

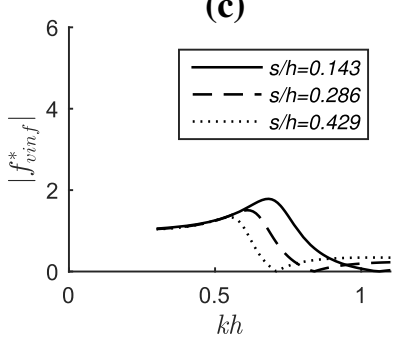

(b)

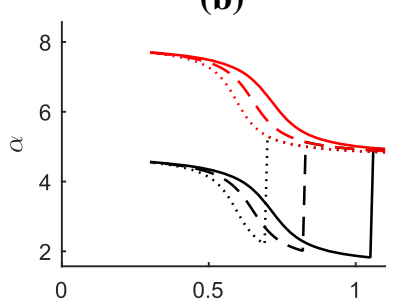

(d)

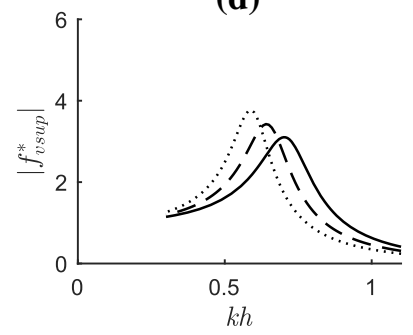

Fig. 9 Amplitude and phase of the force acting on the ballast tank versus $k h$ for $a / h=0.357, b / h=0.429, c / h=0.114$, and $d / h=0.143$ for different values of $s / h$ : (a) amplitude of the total vertical force; (b) phase of the vertical force on the upper face (red lines) and on the lower face (black lines); (c) amplitude of the vertical force on the lower face; (d) amplitude of the vertical force on the upper face

on the ballast tank is proportional to the pressure gradient in the vertical opening times the height $s$ of the ballast tank. However, an increase of $s$ causes a decrease of the maximum force acting on the lower face of the ballast tank.

Figures 5, 6, 7, 8 and 9 show that the force acting on the lower face may vanish for particular values of $k h$. This occurs when the phase of the force acting on the lower face shows 


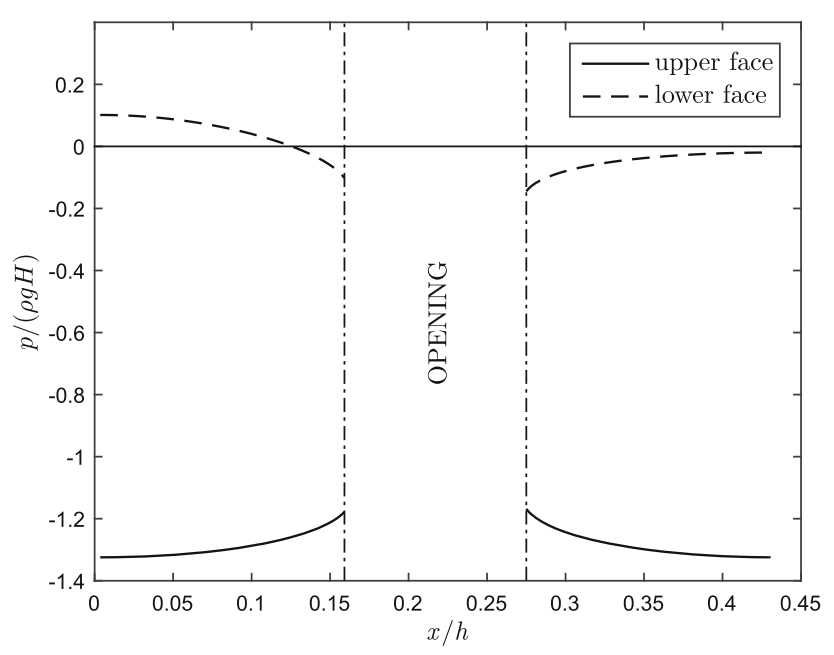

Fig. 10 Dimensionless pressure distribution on the ballast tank faces when the maximum value of $\left|f_{v}^{*}\right|$ is detected: $a / h=0.357, b / h=$ $0.429, c / h=0.114, d / h=0.214, s / h=0.286$, and $k h=0.836$

an abrupt change corresponding to a jump of $\pi$ as it can be observed comparing panels (b) and (c) of Figs. 5, 6, 7, 8 and 9.

Figure 10 shows the pressure distribution on the upper and lower faces of the ballast tank for a configuration giving rise to the vanishing of the vertical force on the lower face at a time during the wave period in which the maximum value of $\left|f_{v}^{*}\right|$ is detected. It can be observed that the pressure on the two sides of the upper face is distributed symmetrically with respect to the axis of the gate. Conversely, the pressure on the lower face is not distributed symmetrically and show both positive and negative values. In these particular conditions these pressures compensate each other giving rise to a vanishing force. The symmetry in the pressure distribution that is observed on the upper face is due to the fact that this area is somewhat shielded from the asymmetries caused by the flow entering from only one side of the structure.

Some of the results shown in the previous figures can find a qualitative explanation through the application of the generalized Bernoulli theorem:

$\frac{1}{g} \frac{\partial V}{\partial t}+\frac{\partial}{\partial r}\left(z+\frac{p}{\varrho g}+\frac{V^{2}}{2 g}\right)=0$,

where $V$ is the magnitude of the velocity and $r$ is the curvilinear abscissa. It should be highlighted that the generalized Bernoulli theorem provides only very rough results which cannot be used for quantitative assessments but only to get qualitative information on the trend of the force. This additional analysis is proposed as a further check on the numerical results and to provide insights into the mechanisms that affect the force. Let us apply the generalized Bernoulli theorem along the path that starts from the point $\left(x=b / 2+s_{1}, z=-h+d / 2\right)$ and ends on the free surface. Neglecting nonlinear terms, as already done in Sect. 2, we get

$\frac{1}{g} \frac{\partial Q}{\partial t}\left(\frac{h-s}{b}-\frac{d}{2 b}+\frac{s}{c}\right)+\eta=\frac{p}{\varrho g}$,

where $Q$ is the flow rate, $\eta$ is the displacement of the free surface with respect to the still water level and $p$ is the dynamic pressure at $\left(x=b / 2+s_{1}, z=-h+d / 2\right)$. It is assumed that the free surface always lies on a horizontal plane. The flow rate $Q$ can be written as $Q=b \partial \eta / \partial t$, therefore

$\frac{\partial \eta}{\partial t}+\sigma_{0}^{2} \eta=\sigma_{0}^{2} \frac{p}{\varrho g}$,

where $2 \pi / \sigma_{0}$ is the natural period and $\sigma_{0}^{2}$ is given by:

$\sigma_{0}^{2}=\frac{g}{h-\frac{d}{2}+s\left(\frac{b}{c}-1\right)}$.

The value of $k h$ corresponding to $\sigma_{0}$ can be computed solving the following equation:

$\frac{1}{1-\frac{d}{2 h}+\frac{s}{h}\left(\frac{b / h}{c / h}-1\right)}=k h \tanh (k h)$

It is expected that the peak of the force occurs when the wave period is close to the natural period of the system. Therefore, the value of $k h$ computed by means of the previous equation is approximately the value of the dimensionless wavenumber at which the peak of the force occurs.

From Eq. (30), it can be observed that when $b / h$ and/or $s / h$ increases, the left hand side decreases, therefore $k h$ decreases. This shows that an increase of $b / h$ or $s / h$ causes the peak of the force to move towards lower values of $k h$. This is just the result shown in Figs. 6 and 9 where it can be observed that when $b / h$ or $s / h$ increases the peak of the force occurs at lower values of $k h$.

Examining once again Eq. (30) it can be observed that when $d / h$ and/or $c / h$ increases the right hand side increases, therefore $k h$ increases and the peak of the force is displaced towards larger values of $k h$. Looking at Fig. 7 and 8 it can be observed that this is just the behaviour of the peak of the force as concerns the effect of $c / h$ and $d / h$. Finally, it can be observed that the parameter $a / h$ does not appear in Eq. (30), thus highlighting that it does not have an important effect. In fact, in the Fig. 5 it can be observed that the parameter $a / h$ has a negligible effect on the position of the force peak.

\section{An expeditious method for estimating the maximum vertical forces due to waves}

The purpose of this section is to provide a tool for estimating the wave load acting on a navigation lock gate, without incur- 

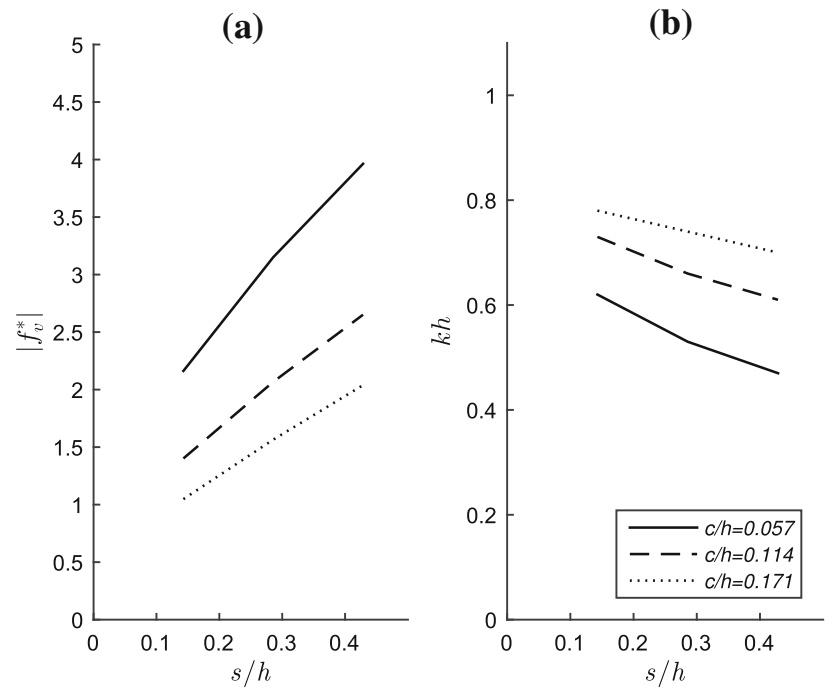

Fig. 11 Maximum dimensionless vertical force (a) and related wavenumber (b) versus relative ballast tank height for $a / h=0.357, b / h=$ 0.429 , and $d / h=0.143$

ring the complications due to the use of the mathematical model described in Sect. 2. The tool is developed exploiting the numerical results of the mathematical model to determine regression functions that link the load to the geometrical and hydrodynamic parameters.

To determine these functions, preliminarily, it is observed that, according to the results of the potential flow model, the relationship between the maximum force $\left|f_{v}^{*}\right|$ and $s / h$ is very close to being linear. This result also applies to the value of $k h$ at which the maximum force is detected and is shown in Fig. 11.

The linearity of $\left|f_{v}^{*}\right|$ with respect to $s / h$ can be justified considering that the force on the ballast thank is proportional to the pressure difference between the upper and the lower face and that this difference can be expressed as the pressure gradient that develops in the vertical opening times $s$.

Based on the results shown in Fig. 11, the maximum dimensionless total vertical force for a fixed geometry and the wave number at which such maximum is detected can be expressed through the following relationships:

$$
\begin{aligned}
& \left|f_{v}^{*}\right|=m_{1} \frac{s}{h}+m_{2} \\
& k h=m_{3} \frac{s}{h}+m_{4}
\end{aligned}
$$

To express the relationship between the coefficients $m_{i}$ and the dimensionless geometrical parameters, the following nonlinear regression is adopted:

$$
\begin{aligned}
m_{i}= & A_{i 0}+A_{i 1} \frac{b}{h}+A_{i 2} \frac{c}{h}+A_{i 3} \frac{d}{h}+A_{i 4} \frac{b}{h} \frac{c}{h} \\
& +A_{i 5} \frac{b}{h} \frac{d}{h}+A_{i 6} \frac{c}{h} \frac{d}{h}
\end{aligned}
$$

Table 2 Coefficient of the non-linear regression

\begin{tabular}{lllll}
\hline$j$ & $A_{1 j}$ & $A_{2 j}$ & $A_{3 j}$ & $A_{4 j}$ \\
\hline 0 & 16.318 & 1.764 & -0.624 & 0.926 \\
1 & -21.660 & 0.643 & -0.313 & -1.470 \\
2 & -82.811 & -18.303 & 5.564 & 2.252 \\
3 & 3.749 & 2.459 & -1.007 & 1.221 \\
4 & 37.718 & 7.907 & -6.074 & -0.408 \\
5 & -6.455 & 0.500 & 0.572 & -1.089 \\
6 & 18.256 & -6.674 & -0.715 & 4.628 \\
7 & 12.991 & -1.577 & 1.143 & 0.980 \\
8 & 167.373 & 40.879 & -3.573 & -6.976 \\
9 & -4.404 & -2.847 & -1.143 & -1.198 \\
\hline & \multicolumn{4}{c}{} \\
& & $A_{i 7}\left(\frac{b}{h}\right)^{2}+A_{i 8}\left(\frac{c}{h}\right)^{2}+A_{i 9}\left(\frac{d}{h}\right)^{2}$ &
\end{tabular}

where a set of coefficients $\left(A_{i 0}, A_{i 1}, \ldots, A_{i 9}\right)$ has been determined for each of the coefficients $m_{1}, m_{2}, m_{3}$ and $m_{4}$ imposing, by means of the least square method, the best fit of Eqs. (31) and (32) to the numerical results provided by the potential flow model. The values of the coefficients $A_{i j}$ are shown in Table 2.

It can be observed that the coefficients characterized by the largest values are those which multiply the ratio $c / h$. On the other hand, the lowest coefficients are those that multiply the ratio $d / h$. These results are consistent with those shown in Sect. 2, which indicate that $c / h$ has a large effect on the force while $d / h$ has only a moderate effect.

The reliability of the regression model, in correctly computing the maximum value of the force and the wave number $k h$ at which such a maximum is detected, is shown in Figs. 12 and 13. It can be observed an excellent agreement between the regression model and the computed values obtained by means of the analytical model described in Sect. 2, both for the dimensionless force $\left|f_{v}^{*}\right|$ and the dimensionless wavenumber $k h$. This agreement is quantified by a root mean square error equal to 0.0435 and 0.0114 for $\left|f_{v}^{*}\right|$ and $k h$ respectively.

\section{Conclusions}

The wave load on a navigation lock sliding gate provided with a vertical front plate and an opening through the ballast tank has been analysed by means of an analytical model based on the linear wave theory.

The results show that the peak values of the dimensionless vertical forces $\left|f_{v}^{*}\right|$ and the value of the wave number $k h$ at which such peak occurs are mainly dependent on the relative width of the opening through the ballast tank $c / h$ and on the relative height of the ballast tank $s / h$. The relative width of 


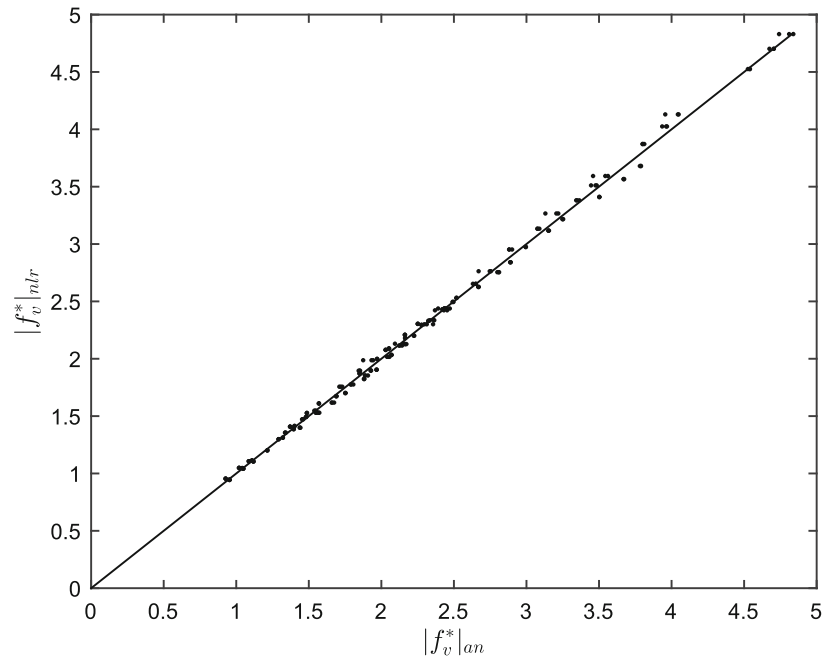

Fig. 12 Maximum dimensionless vertical force: analytical model (an) versus non-linear regression model (nlr)

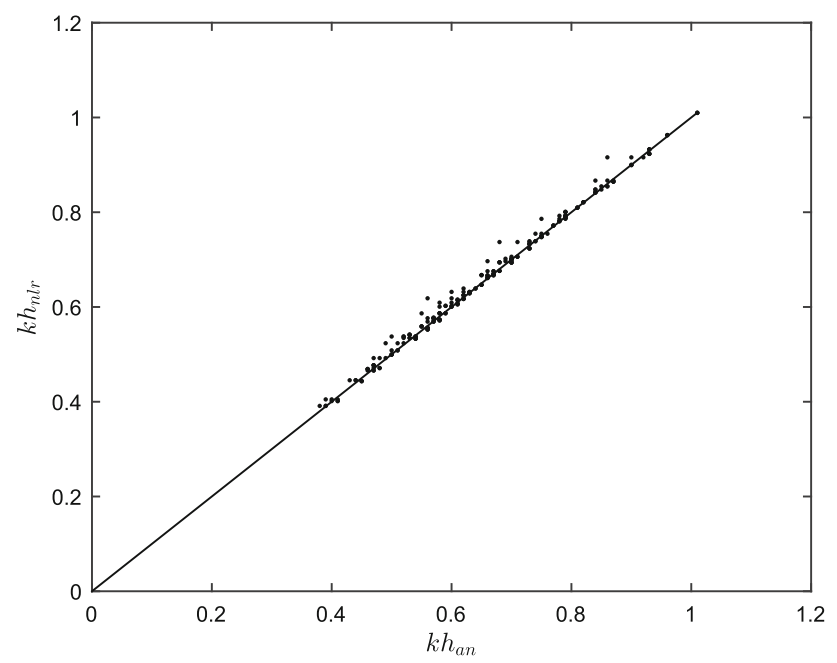

Fig. 13 Wave number at which the maximum dimensionless vertical force: analytical model (an) versus non-linear regression model (nlr)

the ballast tank $b / h$ and the relative height of the slot $d / h$ at the bottom have a moderate effect on the maximum value of the dimensionless vertical force $\left|f_{v}^{*}\right|$ in the range of parameters here considered. However, the ratio $b / h$ has an important influence on the value of the dimensionless wavenumber $k h$ at which the maximum of the force is detected. The relative water depth, $a / h$, above the ballast tank does not show an appreciable influence on the characteristics of the force. The forces acting on the lower and upper faces of the ballast tank can be in phase or out of phase according to the flow parameters. In general, as the dimensionless wave number $k h$ increases the two forces become abruptly in phase, through a jump of $\pi$, when the force acting on the lower face vanishes.

A simple empirical model has been developed which can be used during the pre-design phases of a gate for an expe- ditious evaluation of the maximum vertical force and of the wave number at which this force occurs.

It is highlighted that the proposed analytical model does not include the energy dissipation and the damping of the oscillatory flow. Because of dissipation, the actual forces acting on the gate could be lower than those produced by the model. Therefore, the model provides conservative results.

It is worth emphasizing that the correct evaluation of dissipation requires including the viscous forces in the momentum equation. As a consequence of this inclusion, the flow irrotationality is lost and the governing equations must be solved by means of a numerical approach. In this case it is necessary to adopt large computational grids to describe the flow both in the thin wave boundary layer and in the core region of the flow. This approach involves very high computational costs and is unsuitable for design.

Acknowledgements This work has been partly supported by the project "NEWS - Nearshore hazard monitoring and Early Warning System" (code C1-3.2-60) in the framework of the EU programme INTERREG V-A Italia Malta 2014-2020 and by University of Catania funded projects "Interazione onde-correnti nella regione costiera (INOCS)", "Interazione Moto Ondoso - Strutture (IMOS)", and "VAlutazione del Rischio Idraulico in sistemi cOmplessi (VARIO)".

Funding Open access funding provided by Universitá degli Studi di Catania within the CRUI-CARE Agreement.

Open Access This article is licensed under a Creative Commons Attribution 4.0 International License, which permits use, sharing, adaptation, distribution and reproduction in any medium or format, as long as you give appropriate credit to the original author(s) and the source, provide a link to the Creative Commons licence, and indicate if changes were made. The images or other third party material in this article are included in the article's Creative Commons licence, unless indicated otherwise in a credit line to the material. If material is not included in the article's Creative Commons licence and your intended use is not permitted by statutory regulation or exceeds the permitted use, you will need to obtain permission directly from the copyright holder. To view a copy of this licence, visit http://creativecomm ons.org/licenses/by/4.0/.

\section{References}

Behera H, Ng CO, Sahoo T (2018) Oblique wave scattering by a floating elastic plate over a porous bed in single and two-layer fluid systems. Ocean Eng. 159:280-294. https://doi.org/10.1016/ j.oceaneng.2018.04.031

Cavallaro L, Iuppa C, Scandura P, Foti E (2018) Wave load on a navigation lock sliding gate. Ocean Eng 154:298-310

Fang Q, Hong R, Guo A, Stansby PK, Li H (2018) Analysis of hydrodynamic forces acting on submerged decks of coastal bridges under oblique wave action based on potential flow theory. Ocean Eng. 169:242-252. https://doi.org/10.1016/j.oceaneng.2018.09.031

Guo A, Fang Q, Li H (2015) Analytical solution of hurricane wave forces acting on submerged bridge decks. Ocean Eng. 108:519_ 528. https://doi.org/10.1016/j.oceaneng.2015.08.018

Jalón ML, Lira-Loarca A, Baquerizo A, Losada MA (2018) An analytical model for oblique wave interaction with a partially reflective 
harbor structure. Coastal Eng 143:38-49. https://doi.org/10.1016/ j.coastaleng.2018.10.015

Karmakar D, Soares CG (2012) Oblique scattering of gravity waves by moored floating membrane with changes in bottom topography. Ocean Eng. 54:87-100. https://doi.org/10.1016/j.oceaneng.2012. 07.005

Kundu S, Gayen R, Datta R (2018) Scattering of water waves by an inclined elastic plate in deep water. Ocean Eng. 167:221-228. https://doi.org/10.1016/j.oceaneng.2018.07.054

Liu Y, Li HJ (2013) Wave reflection and transmission by porous breakwaters: a new analytical solution. Coastal Eng 78:46-52. https:// doi.org/10.1016/j.coastaleng.2013.04.003

Liu Y, Yc Li, Teng B (2009) Wave motion over two submerged layers of horizontal thick plates. J. Hydrodyn. 21(4):453-462. https://doi. org/10.1016/S1001-6058(08)60171-7

Losada IJ, Losada MA, Roldán AJ (1992) Propagation of oblique incident waves past rigid vertical thin barriers. Appl Ocean Res 14(3):191-199

Losada MA, Losada IJ, Roldán AJ (1993) Propagation of oblique incident modulated waves past rigid, vertical thin barriers. Appl Ocean Res 15(5):305-310
Malara G, Arena F (2013) Analytical modelling of an u-oscillating water column and performance in random waves. Renew Energy 60:116-126

Monk K, Zou Q, Conley D (2013) An approximate solution for the wave energy shadow in the lee of an array of overtopping type wave energy converters. Coastal Eng 73:115-132. https://doi.org/ 10.1016/j.coastaleng.2012.10.004

Wang KH, Shen Q (1999) Wave motion over a group of submerged horizontal plates. Int. J. Eng Sci. 37(6):703-715. https://doi.org/ 10.1016/S0020-7225(98)00094-9

Wu C, Watanabe E, Utsunomiya T (1995) An eigenfunction expansionmatching method for analyzing the wave-induced responses of an elastic floating plate. Appl. Ocean Res. 17(5):301-310. https://doi. org/10.1016/0141-1187(95)00023-2

Zheng YH, Shen YM, You YG, Wu BJ, Jie DS (2004) On the radiation and diffraction of water waves by a rectangular structure with a sidewall. Ocean Eng. 31(17-18):2087-2104

Publisher's Note Springer Nature remains neutral with regard to jurisdictional claims in published maps and institutional affiliations. 